\title{
mpMRI PI-RADS score 3 lesions diagnosed by reference vs affiliated radiological centers: Our experience in 950 cases
}

\author{
Pietro Pepe ${ }^{1}$, Giuseppe Candiano ${ }^{1}$, Ludovica Pepe $^{1}$, Michele Pennisi ${ }^{1}$, Filippo Fraggetta ${ }^{2}$ \\ ${ }^{1}$ Urology Unit, Cannizzaro Hospital, Catania, Italy; \\ ${ }^{2}$ Pathology Unit, Cannizzaro Hospital, Catania, Italy
}

\begin{abstract}
Summary Introduction: The detection rate for clinically significant prostate cancer (csPCa) in men with mpMRI PI-RADS score 3 diagnosed by affiliated radiology centers vs radiological reference center was evaluated. Materials and methods: From January 2017 to December 2020, 950 men (median age 64 years) underwent mpMRI for abnormal PSA values (median $6.3 \mathrm{ng} / \mathrm{ml}$ ). Among the 950 patients who underwent mpMRI 500 were evaluated by a reference center and 450 by outpatient radiological affiliated centers. All the mpMRI index lesions characterized by a PI-RADS 3 underwent targeted cores combined with extended prostate biopsy. Two radiologists of the radiological reference center revised all the mpMRI lesions 3.

Results: Overall, 361/950 (38\%) patients had a mpMRI lesion PI-RADS score 3: $120 / 500$ cases (24\%) vs 241/450 cases (53.5\%) were diagnosed by reference vs affiliated radiological centers. The detection rate for cT1c csPCa was equal to $26.7 \%$ (35/120 cases) vs 16.6\% (40/241 cases) in men with PI-RADS 3 lesions diagnosed in the reference vs the affiliated radiological centers $(p<0.05)$. Among the 241 PI-RADS score 3 lesions diagnosed by affiliated radiological centers 86/241 (35.7\%) and 36/241 (15\%) were downgraded (PI-RADS scores < 3) and upgraded (PI-RADS score 4) by the dedicated radiologists of the reference center.

Conclusions: In our series, about $35 \%$ and $15 \%$ of PI-RADS score 3 lesions diagnosed by affiliated radiological centers were downgraded and upgraded when revised by experencied radiologists, therefore a second opinion is mandatory especially in men enrolled in active surveillance protocols in whom mpMRI is recommended to reduce the number of scheduled repeated prostate biopsies.
\end{abstract}

KEY WORDS: Prostate cancer; MRI and prostate cancer; PI-RADS score 3; Transperineal targeted biopsy.

Submitted 21 February 2021; Accepted 14 March 2021

\begin{abstract}
INTRODUCTION
Multiparametric magnetic resonance imaging (mpMRI) is strongly recommended before biopsy for the diagnosis of clinically significant prostate cancer (csPCa) (1) in order to reduce the risk of overdiagnosis and to improve the costeffectiveness of prostate biopsy (2). Recently, the European Association of Urology guidelines (3) suggested
\end{abstract}

the evalution of mpMRI combined with clinical parameters (i.e., PSA density, digital rectal examination) instead scheduled prostate biopsies in the reevaluation of men enrolled in Active Surveillance protocols (4-7); therefore, mpMRI quality and radiologist expertice represent a central topic in the decision making for prostate biopsy. The detection rate of csPCa is directly related with the PI-RADS score $(8,9)$ and the results depend on clinical parameters, the number of previous negative biopsies and the quality of targeted mpMRI/TRUS fusion biopsy procedures; the gray zone of mpMRI evaluation is still today represented by the diagnosis of a PI-RADS 3 lesion that could harbour the presence of a clinically significant prostate cancer (csPCa) in about $20-25 \%$ of the cases (1012). At the same time, the number of PI-RADS score 3 diagnosed by radiologists should be limited to a low percentage among all the mpMRI procedures similarly as reported by pathologists for the diagnosis of Atypical Small Cell Acinar Proliferation.

In this study, we report the detection rate for csPCa in men with PIRADS score 3 diagnosed by reference vs affiliated radiological centers.

\section{Patients AND methods}

From January 2017 to December 2020, 950 men (median age 64 years; range: 47-75 years) with negative digital rectal examination underwent mpMRI for abnormal PSA values (median $6.3 \mathrm{ng} / \mathrm{ml}$; range 2.9-102 ng/ml); 680 and 270 underwent initial and repeated prostate biopsy. In 500 men mpMRI was performed at our Hospistal Imaging Department considered aa a reference center; on the contrary, 450 patients were submitted to mpMRI by outpatient radiological affiliated centers.

All mpMRI examinations were previously performed using a 1.5 Tesla scanner equipped with surface 16 channels phased-array coil placed around the pelvic area with the patient in the supine position; multi-planar turbo spin-echo T2-weighted, and axial diffusion weighted imaging, and axial dynamic contrast (ADC) enhanced MRI were performed for each patient (4).

All the mpMRI index lesions characterized by a PI-RADS (version 2) > 3 underwent targeted cores (TPBx: four cores) combined with extended systematic prostate

No conflict of interest declared. 
biopsy (at least 12 cores); the procedure was performed transperineally using a tru-cut 18 gauge needle (Bard; Covington, GA, USA) under sedation and antibiotic prophylaxis (5). The TPBx was done using an Hitachi 70 Arietta ecograph, Chiba, Japan) supplied by a bi-planar trans-rectal probe (13). The data have been collected following the START criteria (14).

Two radiologists of the radiological reference center with 11 years of experience blinded to pre-imaging clinical parameters evaluated the mpMRI data separately and independently. The detection rate for csPCa in men with PIRADS score 3 diagnosed by affiliated vs radiological reference center was evaluated; in addition, all PI-RADS 3 lesions diagnosed in the affiliated radiological centers were revised by the dedicated radiologists and compared with biopsy histology results. For statistical analysis a $p$ value $<0.05$ was considered statistically significant.

\section{RESULTS}

Overall, 361/950 (38\%) patients had a mpMRI lesion PIRADS score 3: $120 / 500$ cases (24\%) vs $241 / 450$ cases (53.5\%) were diagnosed by reference vs affiliated radiology centers, respectively.

The PI-RADS 3 lesions were located in the peripheric and anterior zone of the gland in 190 and 171 cases; moreover, 151 (41.8\%) vs 210 (58.2\%) men underwent cognitive vs fusion targeted biopsy procedure, respectively. The detection rate for Tlc csPCa was equal to $26.7 \%$ (35/120 cases) vs $16.6 \%$ (40/241 cases) in men with PI-RADS 3 lesions diagnosed in the reference vs the affiliated radiological centers $(p<0.05)$ (Table 1): in detail, 24 and 11 vs 25 and 15 of the csPCa were located in the peripheric and anterior zone of the prostate, respectively. None had significant complications (Clavien-Dindo grade I) (15) from prostate biopsy that needed hospital admission.

Table 1.

Quantitative biopsy histology, clinical parameters and ADC values in men with PI-RADS (prostate imaging-reporting and data system) score 3 lesions and clinically significant prostate cancer (csPCa).

\begin{tabular}{|l|c|c|c|}
\hline & $\begin{array}{c}\text { Radiological } \\
\text { reference center }\end{array}$ & $\begin{array}{c}\text { Radiological } \\
\text { affiliated centers }\end{array}$ & P value \\
\hline Overall number of patients with csPCa: $75 / 361$ & $\begin{array}{c}35 / 120 \\
(26.7 \%) \\
\text { csPCa }\end{array}$ & $\begin{array}{c}40 / 241 \\
(16.6 \%) \\
\text { csPCa }\end{array}$ & $<0.05$ \\
\hline Median PSA values (ng/ml) & 8.7 & 9.1 & $>0.05$ \\
\hline Grade group $\quad($ ADC value) & 35 pts & 40 pts & \\
$1 \quad(0.750 \pm 0.162)$ & - & 2 & $<0.05$ \\
$2 \quad(0.635 \pm 0.117)$ & 28 & 19 & $<0.05$ \\
$3 \quad(0.489 \pm 0.093)$ & 17 & 19 & $>0.05$ \\
\hline Median number of positive TPBx cores & 1.5 & 1.0 & $>0.05$ \\
\hline Median number of systematic positive cores & $4(1-9)$ & $5(1-12)$ & $>0.05$ \\
\hline Median GPC (range) & $50 \%$ & $50 \%$ & $>0.05$ \\
\hline Median prostate weight (grams) & 50 & 46 & $>0.05$ \\
\hline Median mpMRI lesion index diameter (mm) & 10 & 9 & $>0.05$ \\
\hline PSA: Prostate specific antigen; TPBx: Tarǵted transperineal fusion biopsy; GPC: Greatest percentage of cancer for \\
single core; ADC: Apparent diffusion coefficient; mpMRl: Multiparametric magnetic resonance imaging; pts: Patients. \\
\hline
\end{tabular}

The clinical parameters (i.e., PSA, weight, PSA density) and the biopsy quantitative histology (i.e., number of positive cores, greatest percentage of cancer and Grade Group) is reported in Table 1; a normal parenchyma was diagnosed in the remaining 286/361 (79.2\%) men.

The median diameter of PI-RADS 3 index lesions was 10 $\mathrm{mm}$. vs $9 \mathrm{~mm}$. in men with csPCa vs normal parenchyma, respectively. Among the 241 PI-RADS score 3 lesions diagnosed by affiliated radiological centers 86/241 (35.7\%) and 36/241 (15\%) were respectively downgraded (PI-RADS scores $<3$ ) and upgraded (PI-RADS score 4) by dedicated radiologist of the reference center. The presence of csPCa was significantly correlated with the ADC value (Table 1).

\section{Discussion}

The improvement of diagnostic imaging by mpMRI has allowed targeted biopsies of the suspicious area, increasing the diagnosis of csPCa and reducing the number of unnecessary systematic biopsy.

Although mpMRI is strongly recommended in men candidate for prostate biopsy (3) or in men enrolled in active surveillance protocols (7), still today, systematic biopsy should be always combined with mpMRI/TRUS fusion biopsy because the increased false negative rate $(5,14)$ of mpMRI (about 20\% of the cases) (6) and the variable diagnostic accuracy of the different mpMRI/TRUS fusion biopsy platforms (17). While the risk of clinically significant in case of PIRADS 4-5 is well established, PIRADS 3 lesions are presented as equivocal and at low risk of aggressive disease with the identification of csPCa is not neglegible $(6,8-11-12)$.

The PI-RADS 3 lesions identified on mpMRI are considered to be "a gray area" in the diagnosis protocol of $\mathrm{PCa}$ $(18,19)$. The main objectives regarding PIRADS 3 score are to accurately diagnose csPCa and avoiding unnecessary biopsies that could have undesirable side effects on patients and thus, avoiding overdiagnosis and overtreatment.

In a recent review, Maggi et al. (20) demonstrated the superiority of combined target and systematic biopsy in detecting csPCa in patients with PIRADS 3 lesions; moreover, they also found that combining PIRADS 3 score with a PSAD > $0.15 \mathrm{ng} / \mathrm{ml} / \mathrm{ml}$ could improve the detection rate of csPCa on prostate biopsy (21).

In addition, to identify the PI-RADS score at high risk for csPCa irrespective of clinical findings many parameters have been reported: index lesion diameter, shape and location of the lesion and the $\operatorname{ADC}$ values $(6,9,11)$. Wu et al. (22) showed that higher ADC values $(0.830 \times 10-3$ $\mathrm{mm}^{2} / \mathrm{sec}$ ) were significantly associated with low-risk prostate cancer; on the contrary, Kim et al. (23) reported a mean $\mathrm{ADC}$ value for $\mathrm{csPCa}$ equal to $(0.741 \pm 0.164)$ $\times 10-3 \mathrm{~mm}^{2} / \mathrm{sec}$.

In our series, the detection rate for $\mathrm{Tlc}$ csPCa was equal to $26.7 \%$ (35/120 cases) vs $16.6 \%$ (40/241 cases) in men with PI-RADS 3 lesions diagnosed in the reference vs the affiliated radiological centers; in detail, among the 241 PI-RADS score 3 lesions diagnosed by affiliated radiological centers $35.7 \%(86 / 241)$ and $15 \%(36 / 241)$ were downgraded (PI-RADS scores < 3 ) and upgraded (PI- 
RADS score 4) by dedicated radiologist of the reference center. In addition, PSA density and ADC value of $0.747 \times 10-3 \mathrm{~mm}^{2} / \mathrm{sec}$, threshold obtained from ROC curve analysis improved the diagnosis for csPCa in the presence of PI-RADS 3 lesions.

Regarding our results some considerations should be made. Firstly, the results were evaluated on biopsy specimens and not on the entire prostate gland or by performing a template mapping biopsy; secondly, although our study represent the real life clinical practice a quality control of the affiliated radiological centers was unknown.

Finally, a greater number of patients and a centralized evaluation of mpMRI results should be performed; moreover, among the 361 men with PI-RADS score 3 only 210 (58.2\%) underwent fusion targeted prostate biopsy.

In conclusion, PI-RADS 3 lesions exhibited aggressive features in a not negligible proportion of cases but a quality control of mpMRI by experienced radiologists improve the accuracy of the procedure; a second opinion is mandatory especially in men enrolled in AS protocols in whom clinical parameters $(5,24,25)$ and mpMRI $(26-$ 28) are recommended to reduce the number of scheduled repeated prostate biopsies.

\section{REFERENCES}

1. Kasivisvanathan V, Rannikko AS, Borghi M, and PRECISION Study Group Collaborators. MRI-targeted or standard biopsy for prostate-cancer diagnosis. N Engl J Med 2018; 378:1767-1777.

2. Pepe P, Pepe G, Pepe L, et al. Cost-effectiveness of multiparametric MRI in 800 men submitted to repeat prostate biopsy: results of a public health model. Anticancer Res. 2018; 38:2395-2398.

3. Mottet N, Cornford P, van der Bergh RCN, et al. EAU Oncology guideline: Prostate Cancer. 2020.

4. Pepe P, Garufi A, Priolo GD, et al. Accuracy of 3 Tesla pelvic phased-array multiparametric MRI in diagnosing prostate cancer at repeat biopsy. Arch Ital Urol Androl. 2014; 4:336-339

5. Pepe P, Pepe L, Pennisi M, Fraggetta F. Which prostate biopsy in men enrolled in Active Surveillance? Experience in 110 men submitted to scheduled three-years transperineal saturation biopsy combined with fusion targeted cores Clin Genitourin Cancer 2021; S1558-7673(21)00027-6.

6. Pepe P, Garufi A, Priolo GD, et al. Is it time to perform only MRI targeted biopsy? Our experience in 1032 men submitted to prostate biopsy. J Urol. 2018; 200:774-778, 2018.

7. Pepe P, Garufi A, Priolo G, and Pennisi M. Can MRI/TRUS fusion targeted biopsy replace saturation prostate biopsy in the re-evaluation of men in active surveillance? World J Urol 2016; 34:12491453.

8. Pepe P, Cimino S, Garufi A, et al. Confirmatory biopsy of men under active surveillance: extended versus saturation versus multiparametric magnetic resonance imaging/transrectal ultrasound fusion prostate biopsy. Scand J Urol. 2017; 51:260-263.

9. Pepe P, Cimino S, Garufi A, et al. Detection rate for significant cancer at confirmatory biopsy in men enrolled in active surveillance protocol: 20 cores vs 30 cores vs vs MRI/TRUS FUSION prostate biopsy. Arch Ital Urol Androl. 2016; 88:300-303.

10. Steinkohl F, Gruber L, Bektic J, et al. Retrospective analysis of the development of PIRADS 3 lesions over time: when is a follow-up MRI reasonable? World J Urol 2018; 36:367-373.

11. Hansen NL, Koo BC, Warren AY, et al. Sub-differentiating equivocal PI-RADS-3 lesions in multiparametric magnetic resonance imaging of the prostate to improve cancer detection. Eur J Radiol. 2017; 95:307-313.

12. Schoots IG. MRI in early prostate cancer detection: how to manage indeterminate or equivocal PI-RADS 3 lesions? Transl Androl Urol. 2018; 7:70-82.

13. Pepe P, Garufi A, Priolo G, and Pennisi M. Transperineal Versus Transrectal MRI/TRUS Fusion targeted biopsy: detection rate of clinically significant prostate cancer. Clin Genitourin Cancer. 2017; e33-e36.

14. Moore CM, Kasivisvanathan V, Eggener S, and START Consortium. Standards of reporting for MRI-targeted biopsy studies (START) of the prostate: recommendations from an international working group. Eur Urol. 2013; 64:544-552.

15. Dindo D, Demartines N and Clavien PA. Classification of surgical complications. A new proposal with evaluation in a Cohort of 6336 patients and results of survey. Ann Surgery. 2004; 2:205-213.

16. Rosenkrantz AB, Verma S, Choyke P, et al. Prostate magnetic resonance imaging and magnetic resonance imaging targeted biopsy in patients with a prior negative biopsy: a consensus statement by AUA and SAR. J Urol. 2016; 196:1613-1618.

17. Westhoff N, Siegel FP, Hausmann D, et al. Precision of MRI/ultrasound-fusion biopsy in prostate cancer diagnosis: an ex vivo comparison of alternative biopsy techniques on prostate phantoms. World J Urol. 2017; 35:1015-1022.

18. van der Leest M, Cornel E, Israël B, et al. Head-to-head comparison of transrectal ultrasound-guided prostate biopsy versus multiparametric prostate resonance imaging with subsequent magnetic resonance-guided biopsy in biopsy-naïve men with elevated prostatespecific antigen: a large prospective multicenter clinical study. Eur Urol. 2019; 75:570-578.

19. Ahmed HU, El-Shater Bosaily A, Brown LC, et al. Diagnostic accuracy of multi-parametric MRI and TRUS biopsy in prostate cancer (PROMIS): a paired validating confirmatory study. Lancet. 2017; 389:815-822.

20. Maggi M, Panebianco V, Mosca A, et al. Prostate imaging reporting and data system 3 ccategory cases at multiparametric magnetic resonance for prostate cancer: a systematic review and meta-analysis. Eur Urol Focus. 2020; 6:463-478.

21. Roscigno M, Stabile A, Lughezzani G, et al. Multiparametric magnetic resonance imaging and clinical variables: Which is the best combination to predict reclassification in active surveillance patients? Prostate Int. 2020; 8:167-172.

22. Wu X, Reinikainen P, Vanhanen A, et al. Correlation between apparent diffusion coefficient value on diffusion-weighted MR Imaging and Gleason score in prostate cancer. Diagn Interv Imaging. 2017; 98:63-71.

23. Kim TH, Kim CK, Park BK, et al. Relationship between Gleason score and apparent diffusion coefficients of diffusion-weighted magnetic resonance imaging in prostate cancer patients. Can Urol Assoc J. 2016;E377-E382.

24. Roscigno M, Stabile A, Lughezzani G, et al. The use of muktiparametric resonance imaging for follow-up patientes included in Active Suirvellance Protocol, can PSA density discriminate patients 
at different risk of reclassification? Clinical Genitourinary Cancer 2020; 18:e698-e704.

25. Pepe P, Dibenedetto G, Pepe L, Pennisi M. Multiparametric MRI vs Select MDX accuracy in the diagnosis of clinically significant $P C a$ in men enrolled in Active Surveillance. In Vivo 2020; 34: 393-396.

26. Pepe P, Davide D'Urso, Garufi A, et al. Multiparametric MRI apparaent diffusion coefficient (ADC) accuracy in diagnosing clinically significant prostate cancer. In Vivo 2017; 31:415-418.
27. Lourenço M, Pissarra P, Vieira D, et al. Lesion location agreement between prostatic multiparametric magnetic resonance, cognitive fusion biopsy and radical prostatectomy piece. Arch Ital Urol Androl. 2020; 91:218-223.

28. Stanzione A, Creta M, Imbriaco M, et al. Attitudes and perceptions towards multiparametric magnetic resonance imaging of the prostate: A national survey among Italian urologists. Arch Ital Urol Androl. 2020; 92:291-296.

\section{Correspondence}

Pietro Pepe, MD (Corresponding Author)

piepepe@hotmail.com

Urology Unit, Cannizzaro Hospital, Via Messina 829, Catania (Italy)

Giuseppe Candiano, MD

urocandia@gmail.com

Ludovica Pepe, MD

ludopepe97@gmail.com

Michele Pennisi, MD

michepennisi2@virgilio.it

Filippo Fraggetta, MD

filippofra@hotmail.com

Pathology Unit, Cannizzaro Hospital, Via Messina 829, Catania (Italy) 